\title{
Influence of combined epidural anesthesia on cognitive function, inflammation and stress response in elderly liver cancer patients undergoing surgery
}

\author{
YANG SU ${ }^{1}$, YANAN PU ${ }^{1}$, ZHENGNAN ZHAO ${ }^{2}$ and XIANGLONG YANG ${ }^{1}$ \\ Departments of ${ }^{1}$ Emergency Anesthesia and ${ }^{2}$ Orthopedics, The Second Affiliated Hospital of \\ Dalian Medical University, Dalian, Liaoning 116027, P.R. China
}

Received October 18, 2019; Accepted December 16, 2019

DOI: $10.3892 / \mathrm{ol} .2020 .11395$

\begin{abstract}
Effects of combined epidural anesthesia on the cognitive function, inflammation and stress response in the elderly liver cancer patients undergoing surgery were explored. Elderly liver cancer patients $(n=100)$ undergoing surgery in the Second Affiliated Hospital of Dalian Medical University from January 2015 to December 2018 were enrolled and randomly divided into observation group $(n=50)$ and control group $(\mathrm{n}=50)$. In control group only conventional anesthesia was performed using $2 \mu \mathrm{g} / \mathrm{kg}$ fentanyl, $1.5 \mathrm{mg} / \mathrm{kg}$ propofol and $0.2 \mathrm{mg} / \mathrm{kg}$ atracurium, in addition to the procedures in the control group, combined epidural anesthesia was administered using $0.5 \%$ bupivacaine for $15 \mathrm{sec}$ and maintained via $0.25 \%$ bupivacaine in the observation group. The anesthetic effect was observed and the arterial oxygen saturation $\left(\mathrm{SaO}_{2}\right)$, heart rate (HR), mean arterial pressure (MAP) and mini-mental state examination (MMSE) and cognitive function scores by cognitive abilities screening instrument (CASI) were evaluated in the patients, and their blood was drawn to detect the inflammatory factors interleukin (IL)-6, IL-1 and tumor necrosis factor- $\alpha$ (TNF- $\alpha$ ), superoxide dismutase (SOD), malondialdehyde (MDA), catalase (CAT), norepinephrine and epinephrine. The observation group exhibited a better anesthetic effect and obviously smaller decreases in the $\mathrm{SaO}_{2}$ and MAP and increase in HR than the control group $(\mathrm{P}<0.05)$. The MMSE and CASI scores, and the content of IL-1, IL-6, TNF- $\alpha$, MDA, CAT, norepinephrine and epinephrine in the observation group was obviously lower than that in the control group $(\mathrm{P}<0.05)$, while the content of SOD was evidently higher than that in the control group $(\mathrm{P}<0.05)$. Overall postoperative
\end{abstract}

Correspondence to: $\mathrm{Dr}$ Xianglong Yang, Department of Emergency Anesthesia, The Second Affiliated Hospital of Dalian Medical University, 467 Zhongshan Road, Shahekou, Dalian, Liaoning 116027, P.R. China

E-mail: maox400@126.com

Key words: combined epidural anesthesia, elderlyliver cancer patients undergoing surgery, cognitive function, inflammation, stress response conditions in the observation group was superior to the control group $(\mathrm{P}<0.05)$, with the incidence rate of cognitive disorder lower than that in the control group $(\mathrm{P}<0.05)$. Combined epidural anesthesia dramatically improves the postoperative conditions and cognitive function and relieve inflammatory and stress responses in the patients with a better anesthetic effect, thus holding promise for application.

\section{Introduction}

Since the life expectancy of humans has been increasing over the past several decades, the management of elderly cancer patients is becoming a global issue. By 2025, the populations aged $\geq 65$ years will account for $20 \%$ of the total in developed countries, and they are at a higher risk of malignant diseases (1). Currently, this population group exhibits $60 \%$ of the newly diagnosed cancers and $70 \%$ death-related cancers in America and Europe. Therefore, the number of elderly tumor patients is expected to steadily increase (2). For example, it is estimated that there will be more elderly patients with liver cancer, a common malignant tumor worldwide. A study found that the incidence rate of complications and the mortality rate are obviously raised in elderly liver cancer patients after hepatectomy (3). It is well known that advanced age is associated with the high rates of comorbidity, especially cardiovascular diseases and aggravated physiological reserves. According to several studies, only $20 \%$ of patients aged $\geq 70$-years undergo hepatectomyfor liver cancer that is currently the second leading cause of cancer-related deaths in males worldwide (4). Although hepatectomy together with other major surgeries has been considered to be high-risk (5), surgical resection should be a preferred treatment regimen for liver cancer according to the studies on some elderly patients $(6,7)$. With the improvement in surgical techniques and perioperative assessment and progress in anesthesia and medical care, hepatectomy has become a safer surgery, even for elderly patients (8).

It may be difficult to defend against liver cancer using optimal treatment strategy in such elderly patients who suffer from diabetes, kidney failure, lung and cardiovascular diseases and other complications or who are exposed to other higher-risk factors. Liver cancer is normally diagnosed in middle-aged and older people, most of whom are accompanied by cirrhosis with 
an incidence rate expected to rise (9). Additionally, the development of vaccines and antiviral therapy has improved the long-term control of chronic hepatitis $\mathrm{B}$ or $\mathrm{C}$, but promoted the incidence of cirrhosis and the progression of liver cancer. Hepatitis $\mathrm{C}$ virus contamination usually occurs in adulthood and causes more severe consequences to elderly patients, such as severe histological damage and more cirrhosis, the latter of which is the leading cause of liver cancer in the elderly (10). Liver cancer patients tend to experience postoperative cognitive dysfunction (POCD) that is a common nervous system complication in the elderly patients after operation (11). POCD serves as a subtle clinical manifestation of dysmnesia and gradual declines in attention and information processing ability and has the main symptoms of anxiety, personality change and memory impairment, which are associated with the extending of hospital stay, decrease in living quality and higher incidence and mortality rates (12). Previous studies have demonstrated that POCD may be attributed to age, surgery and anesthesia (13). Hence, anesthetists have paid special attention to it. There is evidence that the increase in inflammation may be a prediction parameter for POCD (14). Meta analyses manifested that interleukin (IL)- 6 probably acts as a biomarker to guide the prevention and treatment of POCD (15). The over-activation of neutrophils and endothelial cells up-regulates cell surface adhesion molecules including selectins and integrins to promote the adhesion of neutrophils to the inflamed vascular endothelium. The resulting neutrophils are extravasated into vital organs and then degranulate, release proteases and other hydrolases as well as reactive oxygen species and synthesize inflammatory cytokines, which are the key steps in the development and progression of POCD (16). Liver dysfunction may also be induced by inflammatory responses and further stimulates the progression of liver cancer (17). Furthermore, liver cancer patients have more stress responses, of which oxidative stress is related to the high mortality and incidence rates of liver cancer, and the prognosis of liver cancer can be improved using antioxidant stress medications (18).

In the present study, the elderly liver cancer patients were enrolled and after epidural anesthesia, their arterial oxygen saturation $\left(\mathrm{SaO}_{2}\right)$, heart rate (HR) and mean arterial pressure (MAP), mini-mental state examination (MMSE) and cognitive function scores by cognitive abilities screening instrument (CASI), and the indicators, namely the inflammatory factors IL-6, IL-1 and tumor necrosis factor- $\alpha$ (TNF- $\alpha$ ), superoxide dismutase (SOD), malondialdehyde (MDA), catalase (CAT), norepinephrine and epinephrine, were detected, and the postoperative conditions and the incidence of cognitive disorder were compared between the two groups to establish that combined epidural anesthesia can remarkably effectively reduce the incidence rate of cognitive disorder, inflammatory factors and oxidative stress in elderly liver cancer patients and provide theoretical and experimental bases for the preoperative anesthesia and postoperative recovery in liver cancer patients.

\section{Patients and methods}

General information. A total of 100 elderly liver cancer patients undergoing surgery in the Second Affiliated
Hospital of Dalian Medical University (Dalian, China) from January 2015 to December 2018 were selected as the subjects. After their informed consent was obtained, these patients were included into this study and randomly divided into observation group $(n=50)$ and control group $(n=50)$ in accordance with the informed consent. Inclusion criteria: i) patients definitely diagnosed with liver cancer through histological examination of the liver; ii) those with the preoperative MMSE score $>27$ points; iii) those who received no treatment previously; and iv) those who were not allergic to the medications in this study. Exclusion criteria: i) patients with drug allergy; ii) those with severe cardiovascular or cerebrovascular disease; iii) those suffering from secondary infection complicated with severe abnormality in liver or kidney functions; or iv) those with mental disease who were unable to communicate normally. All the clinical specimens in this experiment were collected based on the Declaration of Helsinki and with the approval of the patients and their family members, and the clinical trial regimen was approved by the Ethics Committee of the Second Affiliated Hospital of Dalian Medical University. The patients' clinical information collected at admission are shown in Table I.

Anesthetic methods. In control group, the patients inhaled $2 \mu \mathrm{g} / \mathrm{kg}$ fentanyl, $1.5 \mathrm{mg} / \mathrm{kg}$ propofol and $0.2 \mathrm{mg} / \mathrm{kg}$ atracurium for anesthesia induction and received mechanical ventilation using endotracheal intubation with the tidal volume and respiratory rate controlled properly. During surgery, they were infused continuously with propofol at $5 \mathrm{mg} / \mathrm{kg} / \mathrm{h}$ using an intravenous micropump and with fentanyl for the maintenance of anesthesia, while the atracurium was intermittently infused to keep the muscle relaxed. In the observation group, the patients were given combined epidural anesthesia based on the procedures in the control group: epidural puncture was first performed at the L3-L4 level, and $10 \mathrm{mg}$ of $0.5 \%$ bupivacaine was infused through the subarachnoid space for $\sim 15 \mathrm{sec}$, followed by infusion of $0.25 \%$ bupivacaine via the epidural space and oxygen absorption of patients with face masks for maintenance of anesthesia.

Observation of anesthetic effect in the two groups of patients. The anesthetic effect was assessed according to the international judgment standards: Markedly effective: during surgery, the anesthetized patients are unconscious and relatively quiet and feel no pain, effective: After anesthesia, the patients have vague consciousness and feel mild pain intraoperatively, and ineffective: The patients anesthetized are restless and feel intense pain during operation. The number of each type of cases was recorded in detail to analyze the anesthetic effect.

Comparisons of basic postoperative conditions. After operation, the basic conditions of patients in the two groups were comprehensively evaluated through observing the indicators such as operation duration, sleep time, spontaneous breathing recovery time, total fluid infusion volume and total fluid loss volume, which could indirectly reflect the anesthetic effect.

MMSE and CASI scores and POCD. The cognitive function of patients was scored using the MMSE scale and CASI based on the criteria that the total score is 30 points and the higher the 
Table I. Clinical information of patients.

\begin{tabular}{lcc}
\hline Parameter & Control group & Observation group \\
\hline $\mathrm{n}$ & 50 & 50 \\
Male & 24 & 25 \\
Mean age (years) & $60 \pm 15$ & $61 \pm 18$ \\
Mean weight $(\mathrm{kg})$ & $48 \pm 10.5$ & $50 \pm 12$ \\
BMI $\left(\mathrm{kg} / \mathrm{m}^{2}\right)$ & $22.5 \pm 1.2$ & $21.9 \pm 2.0$ \\
\hline
\end{tabular}

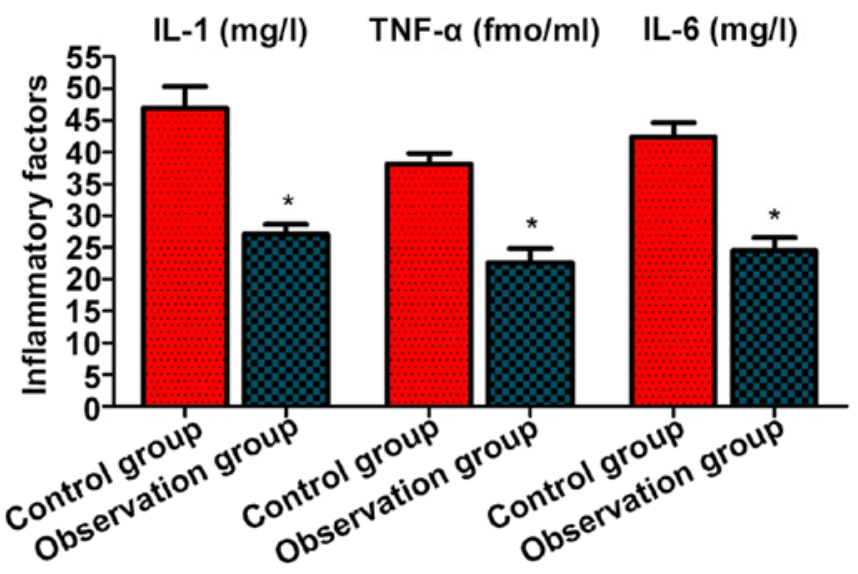

Figure 1. Serum inflammatory factors. Content of IL-6, IL-1 and TNF- $\alpha$ in observation group is substantially lower than those in control group $(\mathrm{P}<0.05)$. ${ }^{*} \mathrm{P}<0.05$ vs. control group. IL, interleukin; TNF- $\alpha$, tumor necrosis factor- $\alpha$.

score is, the better the cognitive function will be. The scores were recorded by 3 nursing staffs in detail, with the mean reflecting the cognitive function of patients, and the number of cognitive dysfunction cases was recorded.

Determination of patients' $\mathrm{HR}, \mathrm{MAP}$ and $\mathrm{SaO}_{2}$. The HR, MAP and $\mathrm{SaO}_{2}$ were determined as follows. Auscultation was conducted at the left ventricle of patients using a stethoscope at resting state, and the heart beats per min were recorded as the HR of patients in triplicate. After the sphygmomanometer was opened, the arm was wrapped up with the cuff, and then the rubber ball was squeezed to read the systolic and diastolic pressure values. Finally, MAP was calculated based on the formula: $\mathrm{MAP}=$ diastolic pressure $+1 / 3$ pulse pressure difference. All the patients were subjected to synchronous intermittent command ventilation (SIMV) via endotracheal intubation in the ward, and the improvement in the clinical symptoms of the patients was observed. After the $\mathrm{SaO}_{2}$ was measured and recorded, the patients were allowed to breathe autonomously.

Detection of serum inflammatory factors via enzyme-linked immunosorbent assay (ELISA). Venous blood (5 ml) was drawn from the arm of the patients, placed in the Eppendorf (EP) tube containing anticoagulant and centrifuged at 2,000 $\mathrm{x} \mathrm{g}$ at room temperature for $15 \mathrm{~min}$. The supernatant was collected to detect serum inflammatory factors IL-6, IL-1 and TNF- $\alpha$ in accordance with the operation instructions of the ELISA kit
(Nanjing Jiancheng Bioengineering Institute). Subsequently, the absorbance in each group was measured using a microplate reader.

Detection of serum oxidative stress indicators. Venous blood $(5 \mathrm{ml})$ was drawn from the arm of the patients, placed in the EP tube containing anticoagulant and centrifuged at $2,000 \mathrm{xg}$ at room temperature for $15 \mathrm{~min}$. The supernatant was collected for detection of changes in serum oxidative stress indicators MDA, SOD and CAT based on the operation instructions of the ELISA kit (Nanjing Jiancheng Bioengineering Institute), followed by measurement of absorbance in each group using the microplate reader.

Determination of norepinephrine and epinephrine in the two groups of patients. Venous blood $(5 \mathrm{ml})$ was collected from the arm of the patients, placed in the EP tube containing anticoagulant and centrifuged at 3,000 $\mathrm{x} g$ at room temperature for $10 \mathrm{~min}$. The supernatant was obtained and then the serum norepinephrine and epinephrine were detected by the person specially assigned using a high-performance liquid chromatography analyzer according to the operation instructions of the instrument.

Statistical analysis. The raw experimental data recorded were processed using SPSS 21.0 analysis software, and subjected to multiple comparisons. The experimental results obtained were expressed as mean \pm standard deviation (mean \pm SD), and $\mathrm{P}<0.05$ indicates statistically significant differences. Histograms were analyzed using GraphPad Prism 5.0.

\section{Results}

Anesthetic effect in the two groups of patients. As shown in Table II, the total effective rates of clinical anesthesia in the observation and control groups were 96 and $86 \%$, respectively, showing a statistically difference $(\mathrm{P}<0.05)$.

Comparisons of basic postoperative conditions. According to Table III, observation group exhibited notably shorter operation duration, sleep time and spontaneous breathing recovery time and markedly smaller total fluid infusion volume and total fluid loss volume than the control group $(\mathrm{P}<0.05)$, and the basic postoperative conditions were better in the observation group.

MMSE and CASI scores and POCD. According to the results (Table IV), observation group had lower MMSE and CASI scores and fewer cases of POCD than the control group $(\mathrm{P}<0.05)$.

HR, MAP and $\mathrm{SaO}_{2}$ in patients. HR and MAP were increased, while $\mathrm{SaO}_{2}$ declined in both groups after operation, but the degree of the increase in HR and MAP and decrease in $\mathrm{SaO}_{2}$ in the observation group were obviously smaller than those in the control group $(\mathrm{P}<0.05)$ (Table $\mathrm{V})$.

Serum inflammatory factors detected via ELISA. As shown in Fig. 1, observation group had remarkably lower content of IL-6, IL-1 and TNF- $\alpha$ than the control group $(\mathrm{P}<0.05)$. 
Table II. Clinical anesthetic effect.

\begin{tabular}{|c|c|c|c|c|}
\hline Group & Markedly effective & Effective & Ineffective & Total effective rate $(\%)$ \\
\hline Control group & 16 & 27 & 7 & 86 \\
\hline Observation group & $24^{\mathrm{a}}$ & $24^{\mathrm{a}}$ & $2^{\mathrm{a}}$ & $96^{\mathrm{a}}$ \\
\hline
\end{tabular}

Anesthetic effect is compared between observation group and control group based on the total effective rate of clinical anesthesia (96 vs. $86 \%$ ), showing a statistically difference $(\mathrm{P}<0.05)$. ${ }^{\mathrm{a}} \mathrm{P}<0.05$ vs. control group.

Table III. Basic postoperative conditions.

\begin{tabular}{|c|c|c|c|c|c|}
\hline Group & $\begin{array}{l}\text { Operation duration } \\
\qquad(\mathrm{min})\end{array}$ & $\begin{array}{l}\text { Sleep } \\
\text { time }\end{array}$ & $\begin{array}{l}\text { Autonomous breathing } \\
\text { recovery time }\end{array}$ & $\begin{array}{l}\text { Total fluid infusion } \\
\text { volume (1) }\end{array}$ & $\begin{array}{l}\text { Total fluid loss } \\
\text { volume (ml) }\end{array}$ \\
\hline Control group & $190 \pm 10.5$ & $220 \pm 5.4$ & $16 \pm 1.5$ & $1.9 \pm 0.1$ & $380 \pm 20.4$ \\
\hline Observation group & $176 \pm 8.1^{\mathrm{a}}$ & $201 \pm 6.8^{a}$ & $11 \pm 1.9^{\mathrm{a}}$ & $1.6 \pm 0.2^{\mathrm{a}}$ & $360 \pm 18.6^{\mathrm{a}}$ \\
\hline
\end{tabular}

${ }^{a} \mathrm{P}<0.05$ vs. control group.

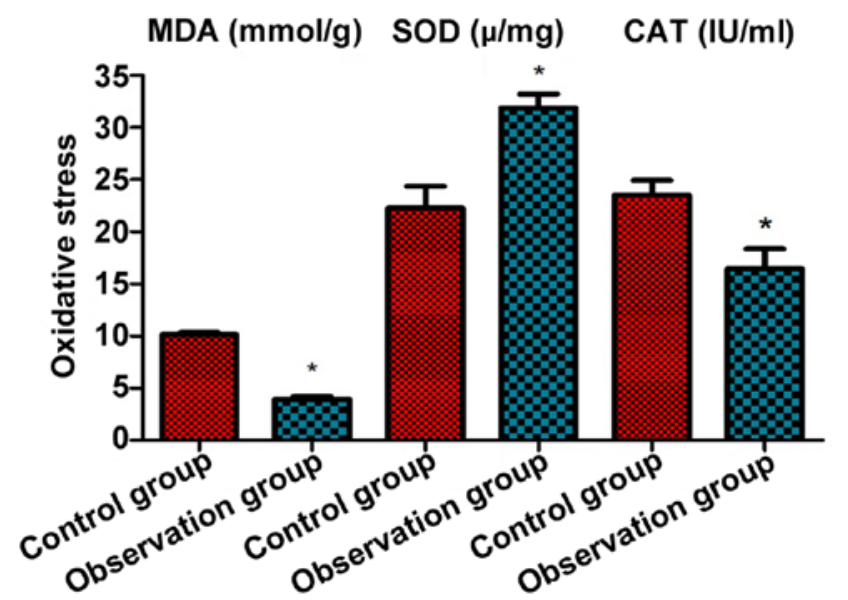

Figure 2. Oxidative stress indicators. In observation group, the content of MDA and CAT declined substantially $(\mathrm{P}<0.05)$, and the opposite tendency was seen for that of SOD $(\mathrm{P}<0.05)$. ${ }^{*} \mathrm{P}<0.05$ vs. control group. MDA, malondialdehyde; CAT, catalase.

Serum oxidative stress indicators detected via ELISA. According to the detection results (Fig. 2), the content of MDA and CAT was considerably lowered $(\mathrm{P}<0.05)$, while that of SOD was remarkably raised in the observation group $(\mathrm{P}<0.05)$.

Norepinephrine and epinephrine in the two groups of patients. As shown in Fig. 3, observation group had remarkably lower content of norepinephrine and epinephrine than control group $(\mathrm{P}<0.05)$.

\section{Discussion}

Hepatectomy is often used to treat malignancies such as liver cancer, cholangiocarcinoma, and metastatic liver cancer. It has been reported (19) that the mortality rate of hepatectomy is lower by several percentiles, but the incidence rate of liver cancer ranges from 20 to $30 \%$ according to another

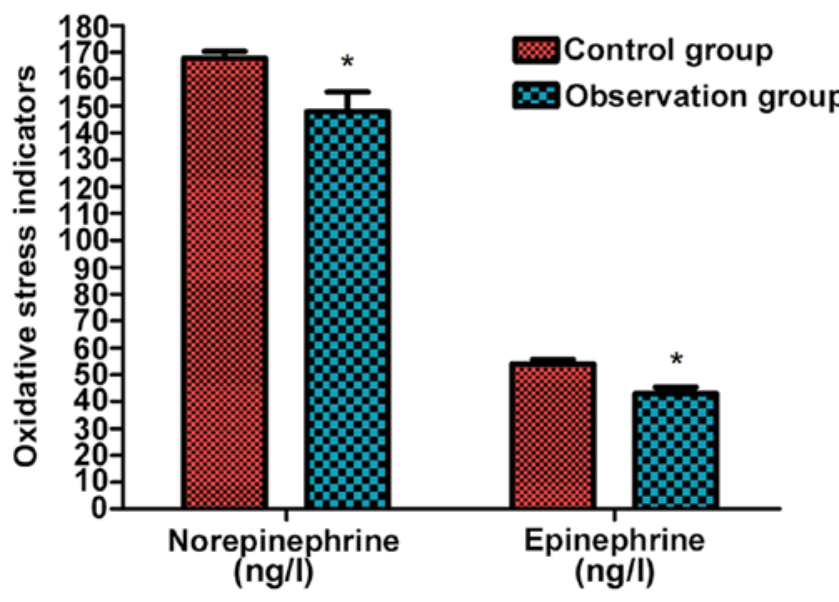

Figure 3. Content of norepinephrine and epinephrine. The content of norepinephrine and epinephrine in observation group is notably lower than that in the control group $(\mathrm{P}<0.05)$. ${ }^{*} \mathrm{P}<0.05$ vs. control group.

report (20). The outcomes of hepatectomy largely depend on the complexity of surgery and liver function of the host as well as anesthesia method. Liver cancer normally originates in the damaged liver. For instance, liver function has been impaired in patients with chronic hepatitis and cirrhosis (21). The young and elderly patients may also differ from each other in the liver histology background and liver function, which is likely to affect the surgical outcomes. Additionally, although metastatic liver tumors are able to progress in normal liver tissues, elderly patients are more susceptible to adverse outcomes probably due to their physiological defects (22). In the present study, the indicators of patients were observed after epidural anesthesia to corroborate that combined epidural anesthesia can effectively reduce the incidence rate of cognitive disorder, inflammatory factors and oxidative stress in elderly liver cancer patients. It was found that the total effective rates of clinical anesthesia in observation and control groups were 96 and $86 \%$, respectively, and that the observation 
Table IV. MMSE and CASI scores and POCD.

\begin{tabular}{lccc}
\hline Group & MMSE score & CASI score & POCD $(\%)$ \\
\hline Before anesthesia & $29.1 \pm 0.5$ & $28.2 \pm 0.4$ & $0 \pm 0$ \\
Control group & $24.2 \pm 0.4$ & $17.3 \pm 0.6$ & $16 \pm 2.0$ \\
Observation group & $27.3 \pm 0.3^{\mathrm{a}}$ & $26.4 \pm 0.2^{\mathrm{a}}$ & $6 \pm 1.5^{\mathrm{a}}$ \\
\hline
\end{tabular}

The MMSE and CASI scores and the incidence rate of POCD in observation group are lower than those in the control group $(\mathrm{P}<0.05)$. ${ }^{a} \mathrm{P}<0.05$ vs. control group. MMSE, mini-mental state examination; CASI, cognitive function scores by cognitive abilities screening instrument; POCD, postoperative cognitive dysfunction.

Table V. HR, MAP and $\mathrm{SaO}_{2}$.

\begin{tabular}{lccc}
\hline Group & $\begin{array}{c}\mathrm{HR} \\
\text { (beats/min) }\end{array}$ & $\begin{array}{c}\mathrm{MAP} \\
(\mathrm{mmHg})\end{array}$ & $\begin{array}{c}\mathrm{SaO}_{2} \\
(\%)\end{array}$ \\
\hline Before anesthesia & $83 \pm 2$ & $81 \pm 4$ & $97 \pm 1$ \\
Control group & $99 \pm 3$ & $95 \pm 5$ & $85 \pm 2$ \\
Observation group & $90 \pm 2^{\mathrm{a}}$ & $86 \pm 2^{\mathrm{a}}$ & $91 \pm 3^{\mathrm{a}}$ \\
\hline
\end{tabular}

The degree of the increase in $\mathrm{HR}$ and MAP and decrease in $\mathrm{SaO}_{2}$ in the observation group are evidently smaller than those in the control group $(\mathrm{P}<0.05)$. ${ }^{\text {a }} \mathrm{P}<0.05$ vs. control group. $\mathrm{HR}$, heart rate; MAP, mean arterial pressure; $\mathrm{SaO}_{2}$, oxygen saturation.

group exhibited notably shorter operation duration, sleep time and spontaneous breathing recovery time, markedly smaller total fluid infusion volume and total fluid loss volume as well as better basic postoperative conditions than the control group. Moreover, the HR and MAP were raised and $\mathrm{SaO}_{2}$ was decreased postoperatively to a smaller degree in the observation group than those in the control group. The above findings are consistent with those in the studies conducted by Nardo et al (23) and Hiraoka et al (24). Previous studies have manifested that the risk of POCD is higher in the patients aged $\geq 60$ years. Bedford (25) reported that $25.8 \%$ of elderly patients have POCD at 1 week after operation, while $9.9 \%$ of patients suffer from POCD at 3 months after operation. Anesthesia may be one of inducers of POCD as well. Different from general anesthesia, local anesthesia has no potential advantage in lowering the incidence rate of POCD (26). Some studies have found that $31 \%$ of the elderly patients undergoing elective surgery experience POCD at $24 \mathrm{~h}$ after the surgery. Another study revealed that epidural anesthesia can lower the incidence rate of POCD to a great extent (27). In the present study, the cognitive function of patients was scored using the MMSE scale and CASI. Based on the criterion that the higher the scores are, the better the cognitive function will be. It was found that the observation group had remarkably lower MMSE and CASI scores and incidence rate of POCD than the control group, suggesting that combined epidural anesthesia is more effective for the postoperative recovery of cognitive function in the patients.
Over the past few years, inflammatory cytokines, which are stress-inducing factors accepted more easily, have attracted extensive attention as the mediators of the perioperative responses for surgeries (28). TNF- $\alpha$ is one of the main cytokines mediating the early-stage responses to tissue damage, and its release can stimulate the production of IL-6. Under normal conditions, IL-6 has a relatively low concentration and even fails to be detected in healthy subjects. Within 30-90 min after operation, the concentration of IL-6 starts rising at the skin incision, which is a sensitive indicator of tissue damage at early stages and the changes in the concentration are associated with duration of surgery and severity of surgical trauma. Furthermore, the increases in TNF- $\alpha$ and IL-6 have been proven to be associated with higher morbidity rates (29). It has been proposed that the increase in oxidative stress is the underlying mechanism of liver dysfunction in liver cancer patients. Oxygen radicals are involved in the pathological damage and the subsequent recovery in liver cancer patients, in which the effect of SOD extensively present can be resisted by MDA, producing cytotoxicity (30). According to the findings in the present study, observation group had remarkably lower content of IL-6, IL-1, TNF- $\alpha$, MDA, CAT, norepinephrine and epinephrine, but substantially higher content of SOD than control group, implying that the application of epidural anesthesia can effectively reduce the inflammatory factors and alleviate oxidative stress in liver cancer patients, which agree with those of previous studies $(31,32)$. All the above-mentioned studies have corroborated that combined epidural anesthesia can lower the incidence rate of cognitive dysfunction, reduce inflammatory factors and ameliorate oxidative stress, with marked effectiveness, which is worthy of further application.

In conclusion, the present study proves that combined epidural anesthesia can effectively reduce the incidence rate of cognitive disorder, decrease inflammatory factors, and improve oxidative stress in elderly liver cancer patients to a great extent through postoperatively observing the cognitive function, secretion of inflammatory factors and stress response in the liver cancer patients given epidural anesthesia, which provides theoretical and experimental bases for preoperative anesthesia and postoperative recovery in liver cancer patients.

\section{Acknowledgements}

Not applicable.

\section{Funding}

No funding was received.

\section{Availability of data and materials}

The datasets used and/or analyzed during the current study are available from the corresponding author on reasonable request.

\section{Authors' contributions}

YS wrote the manuscript. YS and YP collected and analyzed general data of patients. ZZ performed ELISA. YS and XY 
were responsible for statistical analysis. All authors read and approved the final manuscript.

\section{Ethics approval and consent to participate}

The study was approved by the Ethics Committee of the Second Affiliated Hospital of Dalian Medical University (Dalian, China) and informed consents were signed by the patients and/or the guardians.

\section{Patient consent for publication}

Not applicable.

\section{Competing interests}

The authors declare that they have no competing interests.

\section{References}

1. Quaglia A, Tavilla A, Shack L, Brenner H, Janssen-Heijnen M, Allemani C, Colonna M, Grande E, Grosclaude P and Vercelli M; EUROCARE Working Group: The cancer survival gap between elderly and middle-aged patients in Europe is widening. Eur J Cancer 45: 1006-1016, 2009.

2. Aapro MS, Köhne CH, Cohen HJ and Extermann M: Never too old? Age should not be a barrier to enrollment in cancer clinical trials. Oncologist 10: 198-204, 2005.

3. Yamamoto K, Takenaka K, Matsumata T, Shimada M, Itasaka H, Shirabe K and Sugimachi K: Right hepatic lobectomy in elderly patients with hepatocellular carcinoma. Hepatogastroenterology 44 514-518, 1997.

4. Pawlik TM, Schulick RD and Choti MA: Expanding criteria for resectability of colorectal liver metastases. Oncologist 13: 51-64, 2008.

5. Zacharias T, Jaeck D, Oussoultzoglou E, Bachellier P and Weber JC: First and repeat resection of colorectal liver metastases in elderly patients. Ann Surg 240: 858-865, 2004.

6. de Liguori Carino N, van Leeuwen BL, Ghaneh P, Wu A, Audisio RA and Poston GJ: Liver resection for colorectal liver metastases in older patients. Crit Rev Oncol Hematol 67: 273-278, 2008.

7. Simmonds PC, Primrose JN, Colquitt JL, Garden OJ, Poston GJ and Rees M: Surgical resection of hepatic metastases from colorectal cancer: A systematic review of published studies. Br J Cancer 94: 982-999, 2006.

8. Hanazaki K, Kajikawa S, Shimozawa N, Shimada K, Hiraguri M, Koide N, Adachi W and Amano J: Hepatic resection for hepatocellular carcinoma in the elderly. J Am Coll Surg 192: 38-46, 2001.

9. Xu XS, Chen W, Miao RC, Zhou YY, Wang ZX, Zhang LQ, Qu K, Pang Q, Wang RT and Liu C: Survival analysis of hepatocellular carcinoma: A comparison between young patients and aged patients. Chin Med J (Engl) 128: 1793-1800, 2015.

10. Kondo K, Chijiiwa K, Funagayama M, Kai M, Otani K and Ohuchida J: Hepatic resection is justified for elderly patients with hepatocellular carcinoma. World J Surg 32: 2223-2229, 2008.

11. Monk TG, Weldon BC, Garvan CW, Dede DE, van der Aa MT, Heilman KM and Gravenstein JS: Predictors of cognitive dysfunction after major noncardiac surgery. Anesthesiology 108: 18-30, 2008.

12. Cibelli M,Fidalgo AR, Terrando N,MaD,MonacoC,Feldmann M, Takata M, Lever IJ, Nanchahal J, Fanselow MS, et al: Role of interleukin-1beta in postoperative cognitive dysfunction. Ann Neurol 68: 360-368, 2010.
13. Rasmussen LS: Postoperative cognitive dysfunction: Incidence and prevention. Best Pract Res Clin Anaesthesiol 20: 315-330, 2006.

14. Fidalgo AR, Cibelli M, White JP, Nagy I, Maze M and Ma D: Systemic inflammation enhances surgery-induced cognitive dysfunction in mice. Neurosci Lett 498: 63-66, 2011.

15. Peng L, Xu L and Ouyang W: Role of peripheral inflammatory markers in postoperative cognitive dysfunction (POCD): A meta-analysis. PLoS One 8: e79624, 2013.

16. Tsukamoto T, Chanthaphavong RS and Pape HC: Current theories on the pathophysiology of multiple organ failure after trauma. Injury 41: 21-26, 2010.

17. Sato H, Tanaka T, Kita T and Tanaka N: A quantitative study of lung dysfunction following haemorrhagic shock in rats. Int J Exp Pathol 91: 267-275, 2010.

18. Song J, Park J, Kim JY, Kim JD, Kang WS, Muhammad HB, Kwon MY, Kim SH, Yoon TG, Kim TY, et al: Effect of ulinastatin on perioperative organ function and systemic inflammatory reaction during cardiac surgery: A randomized double-blinded study. Korean J Anesthesiol 64: 334-340, 2013.

19. Portolani N, Baiocchi GL, Coniglio A, Tiberio GA, Prestini K, Gheza F, Benetti A and Maria Giulini S: Limited liver resection: A good indication for the treatment of hepatocellular carcinoma in elderly patients. Jpn J Clin Oncol 41: 1358-1365, 2011.

20. Di Benedetto F, Berretta M, D'Amico G, Montalti R, De Ruvo N, Cautero N, Guerrini GP, Ballarin R, Spaggiari M, Tarantino G, et al: Liver resection for colorectal metastases in older adults: A paired matched analysis. J Am Geriatr Soc 59: 2282-2290, 2011.

21. Mizuguchi T, Kawamoto M, Meguro M, Nakamura Y, Ota S, Hui TT and Hirata K: Prognosis and predictors of surgical complications in hepatocellular carcinoma patients with or without cirrhosis after hepatectomy. World J Surg 37: 1379-1387, 2013.

22. Mizuguchi T, Kawamoto M, Meguro M, Hui TT and Hirata K: Preoperative liver function assessments to estimate the prognosis and safety of liver resections. Surg Today 44: 1-10, 2014.

23. Nardo B, Serafini S, Ruggiero M, Grande R, Fugetto F, Zullo A, Novello M, Rizzuto A, BonaiutoE, Vaccarisi S, et al: Liver resection for metastases from colorectal cancer in very elderly patients: New surgical horizons. Int J Surg 33 (Suppl 1): S135-S141, 2016.

24. Hiraoka M, Miyagawa T, Kobayashi H, Takahashi T, Kishi H, Kobayashi $\mathrm{H}$ and Lee I: Successful introduction of modified dorsolumbar epidural anesthesia in a bovine referral center. J Vet Sci 8: 181-184, 2007.

25. Bedford PD: Adverse cerebral effects of anaesthesia on old people. Lancet 269: 259-263, 1955.

26. Steinmetz J, Christensen KB, Lund T, Lohse N and Rasmussen LS; ISPOCD Group: Long-term consequences of postoperative cognitive dysfunction. Anesthesiology 110: 548-555, 2009.

27. Chen J, Yan J and Han X: Dexmedetomidine may benefit cognitive function after laparoscopic cholecystectomy in elderly patients. Exp Ther Med 5: 489-494, 2013.

28. Soop M, Nygren J, Thorell A and Ljungqvist O: Stress-induced insulin resistance: Recent developments. Curr Opin Clin Nutr Metab Care 10: 181-186, 2007.

29. Desborough JP: The stress response to trauma and surgery. Br J Anaesth 85: 109-117, 2000.

30. Brøchner AC and Toft P: Pathophysiology of the systemic inflammatory response after major accidental trauma. Scand J Trauma Resusc Emerg Med 17: 43, 2009.

31. Hadimioglu N, Ulugol H, Akbas H, Coskunfirat N, Ertug Z and Dinckan A: Combination of epidural anesthesia and general anesthesia attenuates stress response to renal transplantation surgery. Transplant Proc 44: 2949-2954, 2012.

32. Li Y, He R, Chen S and Qu Y: Effect of dexmedetomidine on early postoperative cognitive dysfunction and peri-operative inflammation in elderly patients undergoing laparoscopic cholecystectomy. Exp Ther Med 10: 1635-1642, 2015.

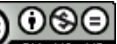

This work is licensed under a Creative Commons Attribution-NonCommercial-NoDerivatives 4.0 International (CC BY-NC-ND 4.0) License. 